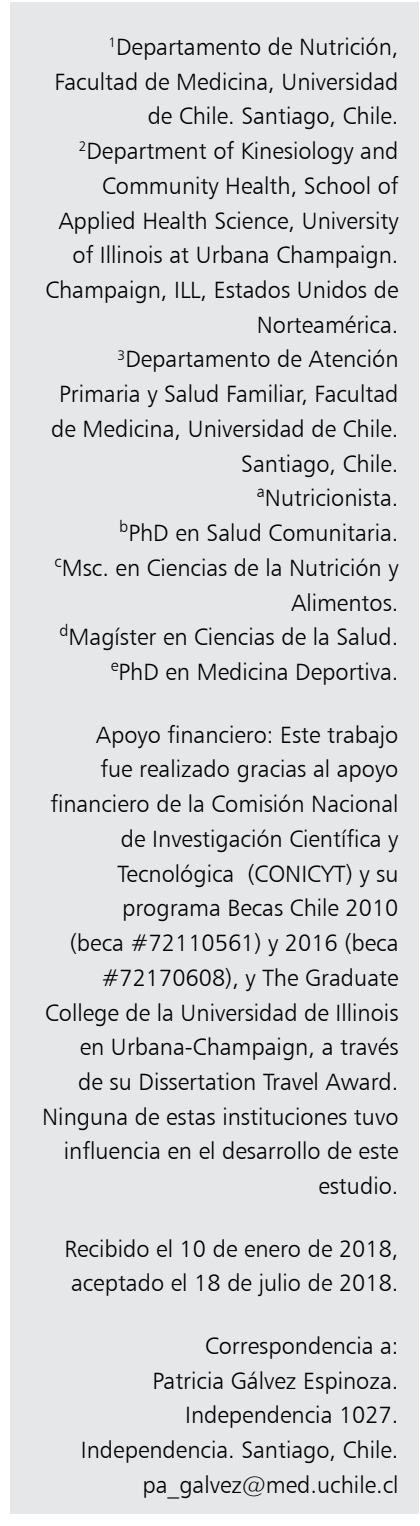

\section{“Comemos así porque...". Entendiendo las conductas alimentarias de mujeres chilenas de bajos recursos}

\author{
PATRICIA GÁLVEZ-ESPINOZA ${ }^{1, \mathrm{a}, \mathrm{b}}$, LORENA IGLESIAS-VEJAR ${ }^{1, \mathrm{a}}$, \\ MARCELA VIZCARRA-CATALÁN ${ }^{2, a, c}$, ANA MARÍA PALOMINO ${ }^{1,3, a, c,}$, \\ ALEJANDRA VALENCIA ${ }^{1, a, d}$, ANDIARA SCHWINGEL ${ }^{2, e}$
}

Background: The prevalence of obesity is higher in women than in men, especially in those of lower socioeconomic status. It is established that this group tends to have a less healthy diet. Aim: To explore the eating behaviors of low-income Chilean women. Material and Methods: Semi-structured interviews with photo-elicitation were conducted with 31 Chilean women aged $36 \pm 6$ years, who lived with a partner and had at least one sibling under 12 years of age. The interviews were transcribed and an inductive analysis of the participants' discourses was made. Results: Seven themes were described in relation to their influence on three eating behaviors (food purchase, preparation and intake): Family, preferences, temporality, financial issues, special occasions, perceptions about food, and availability of food. The family exerted a strong influence on the behaviors and had an impact on the other themes encountered. Conclusions: Eating behaviors were influenced by several factors, both at the personal and environment levels. These factors interacted with each other to produce different forms of behavior modulation. It is therefore problematic to promote healthy eating behaviors while not considering all the influences on this behavior.

(Rev Med Chile 2018; 146: 882-889)

Key words: Feeding Behavior; Qualitative Research; Women.

\section{L} a salud de la mujer es asociada comúnmente a morbilidad del embarazo, parto y puerperio, a VIH, menopausia, entre otros. Menos frecuente pensamos en cómo la obesidad las está afectando, incluso en mayor medida que a los hombres ${ }^{1}$. En América Latina, la prevalencia de obesidad en mujeres es 10 puntos porcentuales más alta que en los hombres en más de 20 países $^{2}$, describiéndose una feminización de la obesidad ${ }^{3}$. En Chile, la prevalencia de obesidad en mujeres mayores de 15 años alcanza a 38,4\%, 8 puntos porcentuales más que en los varones, siendo aquellas en edad fértil y más desfavorecidas económicamente las con mayor prevalencia $(47 \%)^{4,5}$. Dado que las mujeres tienen un rol considerado crítico para el bienestar de la familia ${ }^{6}$, la prevención y tratamiento de la obesidad y sus consecuencias es esencial para evitar el alto costo de este problema de salud para la familia y la sociedad. 
Entonces, resulta interesante cuestionar cuál es la dieta en estas mujeres y cuáles son las causas de esta, especialmente en los segmentos más vulnerables. En general, se sabe que las mujeres de bajos recursos tienden a tener dietas menos saludables. Estudios en Norteamérica y Europa muestran que ellas tienden a tener una dieta con altos niveles de grasa, sodio, calorías y colesterol, combinada con baja ingesta de fibra ${ }^{8}$, además, compran frecuentemente alimentos en lugares de comida rápida9. En América Latina se han encontrado resultados similares. Un estudio en mujeres mexicanas de bajo nivel educacional de zonas rurales mostró que consumían una dieta baja en frutas, verduras y lácteos y un frecuente consumo de refrescos azucarados ${ }^{10}$. Otro estudio, en Brasil, mostró que aquellas mujeres más pobres consumían una dieta que los autores denominaron de "alto riesgo"11. En Chile, Pereira y Erazo destacaron una baja adherencia a las recomendaciones alimentarias en mujeres atendidas en el sistema público de salud chileno ${ }^{12}$. Si bien, existe evidencia respecto a qué comen las mujeres, son muy escasos los estudios en América Latina respecto a las causas que ellas tienen para consumir este tipo de dietas. Contar con esta información podría contribuir a generar intervenciones más focalizadas en este grupo y, así, poder cambiar dichas conductas, favoreciendo una alimentación saludable.

El propósito de este estudio es contribuir a la comprensión de conductas alimentarias en mujeres vulnerables, explorando, a través de un enfoque cualitativo que utiliza photo-elicitation, las razones por las cuales, mujeres chilenas de bajos recursos tienen dietas que podrían contribuir al desarrollo de la obesidad.

\section{Material y Métodos}

Estudio cualitativo con photo-elicitation. Dada su mayor difusión en idioma inglés, es que en este manuscrito utilizaremos photo-elicitation para referirnos a esta técnica. Esta es una técnica que tiene mayor uso en ciencias sociales, pero dada su utilidad, investigadores la han comenzado a usar en salud y en estudios de alimentación y nutrición ${ }^{13,14}$. Photo-elicitation es una técnica participativa definida como la inclusión de imágenes durante entrevistas, como fuentes de estímulo para obtener información ${ }^{15}$, facilitando la verbalización ${ }^{14}$ y la relación investigadorparticipante ${ }^{13}$. Es una técnica participativa como lo es el photo-voice, diferenciándose de este, por su carácter individual y su objetivo de recabar información, más que buscar soluciones ${ }^{15}$.

Este protocolo fue aprobado por el Comité de Ética de la Facultad de Medicina de la Universidad de Chile y por el Institutional Review Board de la Universidad de Illinois de Urbana Champaign.

\section{Participantes}

Mujeres chilenas residentes de áreas urbanas de Santiago-Chile reclutadas por muestreo intenciona ${ }^{16}$ en centros de salud y organizaciones comunitarias. Los criterios de inclusión fueron tener al menos un niño menor de 12 años, vivir con pareja, en un barrio urbano de vulnerabilidad alta o medio-alta, definido por el índice de prioridad social de Chile $^{17}$. Cincuenta mujeres fueron invitadas a participar, 31 terminaron el estudio. Las características de las participantes se muestran en la Tabla 1.

\section{Recopilación de información}

Realizamos entrevistas individuales con photoelicitation entre noviembre de 2014 y marzo de 2015. Una vez firmado el consentimiento de su participación, se les entregó una cámara fotográfica desechable. Se les solicitó tomar 20 fotos de cualquier aspecto en sus vidas que fuera importante para ellas y que estuviera relacionado con alimentación o nutrición, durante 7 días consecutivos. Luego, devolvieron las cámaras y revelamos las imágenes.

\section{Tabla 1. Características generales} de las participantes

\begin{tabular}{|c|c|}
\hline Característica & Valor \\
\hline Edad en años (promedio \pm DS) & $36,2 \pm 6,3$ \\
\hline Al menos 12 años de educación formal (\%) & 74,5 \\
\hline Desempleada (\%) & 61,3 \\
\hline $\begin{array}{l}\text { Ingreso mensual (US\$ per cápita) } \\
\text { (promedio } \pm \text { DS) }\end{array}$ & $166 \pm 124,3$ \\
\hline Seguro de salud público (\%) & 90,3 \\
\hline Presencia de enfermedad crónica (\%) & 35,5 \\
\hline Con sobrepeso u obesidad (\%) & 71 \\
\hline
\end{tabular}


Durante la entrevista, las participantes revisaron sus fotografías y seleccionaron cinco a siete que más deseaban compartir o que consideraban interesantes. Posteriormente, el entrevistador realizó las preguntas sugeridas por la técnica $\mathrm{SHOWeD}{ }^{18}$, incentivando a hablar libremente sobre cada fotografía. La metodología detallada ha sido publicada previamente ${ }^{19}$.

Se logró la saturación teórica de la información ${ }^{20}$ en la entrevista número 20.

\section{Análisis de la Información}

Utilizamos un enfoque inductivo para el análisis de la información. Cinco investigadores seleccionaron 10 entrevistas aleatoriamente, con el objetivo de familiarizarse con la información y buscar códigos iniciales ${ }^{21}$. Posteriormente, el equipo comparó los códigos, reteniendo solo aquellos códigos que la mayoría de los investigadores identificaron y que se acordó unánimemente. Entonces, se creó un libro de códigos general, con el que cada investigador codificó y organizó los datos de las 31 entrevistas, usando Nvivo 10. Una vez codificadas todas las entrevistas, los investigadores revisaron y categorizaron los códigos, para finalmente obtener los temas principales ${ }^{21}$. Además, se discutió el tipo de relaciones o interacciones existentes dentro de cada tema y entre los temas. Ejemplos de citas directas desde las entrevistas son utilizadas para ejemplificar cada tema (Tabla 2), identificando su origen a través de códigos.

\section{Resultados}

Identificamos siete temas que describían cómo las mujeres fundamentaban tres conductas alimentarias: 1) la compra; 2) la preparación y 3) la ingesta de alimentos. Los temas fueron descritos como factores que influencian estas tres conductas alimentarias. La Figura 1 esquematiza cómo las conductas y sus factores interactúan entre sí.

\section{La familia: "Todo se relaciona con ellos..."}

La familia aparece como un factor determinante en las decisiones relacionadas con las conductas alimentarias, influyendo, además, en otros factores, por lo que se ha destacado en la Figura 1. Lo primero que destacó fue la dificultad para separar la discusión sobre la propia alimentación de aquella de sus familias. Ellas generalmente hablaban de "nosotros" (refiriéndose a su familia más cercana) para responder sobre su propia dieta.

Los esposos/parejas e hijos(as) destacaron como grandes influyentes en su dieta. Ellas sabían exactamente lo que su esposo y sus hijos querían o podrían necesitar, tratando de satisfacer esos requerimientos, así como las preferencias alimentarias familiares. En este sentido, ellas mencionaron que compraban, preparaban o comían lo que su núcleo familiar quería, a veces dejando de lado sus propias necesidades.

\section{Las preferencias: "Lo como porque me gusta"}

Las preferencias determinaban lo que ellas comían o cómo preparaban los alimentos. La selección de alimentos dependía de lo que les gustaba en cuanto al sabor, textura o métodos de cocción.

Aunque indicaron tener sus preferencias personales por alimentos o métodos de cocción, su familia influenciaba en que ellas pudieran cumplir con estas, indicando que evitaban cocinar algunas comidas de su gusto porque sabían que a sus familiares no les gustaría. Dado que las participantes especificaban que no podían cocinar dos comidas diferentes, preferían cocinar y comer lo que sabían que su familia comería, dejando sus gustos o preferencias de lado.

\section{Temporalidad: "Es debido al día o época del año"}

La temporalidad presentó dos dimensiones. Primero, se constató que se produce un cambio en las conductas alimentarias de acuerdo al día de la semana; ellas compraban, preparaban y comían de manera diferente entre días de semana y fines de semana. En este último, comían de manera "más especial", incluyendo alimentos fritos, refrescos o pasteles, debido a que en estos días están todos los miembros de la familia en casa, tornándose los tiempos de comida en momentos de reunión familiar.

Segundo, se observó que existe un cambio en la compra e ingesta de alimento, de acuerdo a las estaciones del año. Esta diferencia fue notoriamente expresada entre invierno y verano. Durante el invierno, reducían la ingesta de verduras o frutas crudas señalando que estas les "hacían sentir frío", consumiendo alimentos más calóricos y de mayor temperatura, lo que se revierte durante el verano. Además, durante 
Tabla 2. Citas ejemplificadoras de los temas encontrados

\begin{tabular}{|c|c|}
\hline Tema & Citas \\
\hline \multirow[t]{2}{*}{$\begin{array}{l}\text { Familia "Todo se } \\
\text { relaciona con ellos..." }\end{array}$} & $\begin{array}{l}\text { "[A la hora del té] no tomamos té, tomamos jugo... en vez de bebida y siempre rojo... nos } \\
\text { gusta e hicimos aliado [sándwich de jamón y queso]" (P1) }\end{array}$ \\
\hline & $\begin{array}{l}\text { "...yo estoy acostumbrada... hago el almuerzo, lo sirvo, almuerzo rápido porque tengo que } \\
\text { darle almuerzo a las más chicas que son las que más se demoran en comer... para que no se } \\
\text { les enfríe la comida..." (MI32) }\end{array}$ \\
\hline $\begin{array}{l}\text { Preferencias: "Lo como } \\
\text { porque me gusta" }\end{array}$ & $\begin{array}{l}\text { "...lo que no he comido hace tiempo es el cochayuyo [tipo de alga marina]..., porque a mis } \\
\text { hijos no les gusta... pero tengo ganas de hacer charquicán de cochayuyo, me encanta... pero } \\
\text { no he hecho por lo mismo... porque mis hijos no van a comer" (J19) }\end{array}$ \\
\hline \multirow[t]{2}{*}{$\begin{array}{l}\text { Temporalidad: "Es debido } \\
\text { al día o época del año" }\end{array}$} & $\begin{array}{l}\text { "En los fines de semana, preparo cosas más ricas como papas salteadas, papas fritas... una } \\
\text { vez a la semana preparo cosas fritas, carne asada... a veces hago sushi... algo diferente [que } \\
\text { durante los días de semana]" (MC26) }\end{array}$ \\
\hline & $\begin{array}{l}\text { "En el invierno se consume menos fruta, se compran manzanas, naranjas, mandarinas y de } \\
\text { repente plátanos... pero no las otras frutas, porque no están... en este tiempo [verano] hay } \\
\text { más fruta. Hay más variedad, entonces se va más rápido, porque comen [los miembros de su } \\
\text { familia] más fruta...." (I15) }\end{array}$ \\
\hline \multirow[t]{2}{*}{$\begin{array}{l}\text { Percepciones acerca } \\
\text { de los alimentos: "Este } \\
\text { alimento es..." }\end{array}$} & $\begin{array}{l}\text { "... a mí me da sed y trato de tomar agua... no tomo ni bebida ni jugo, porque ya, siento, como } \\
\text { que ya me mentalicé que la bebida no es para la sed, la bebida es para engordar, entonces } \\
\text { ahora tomo agua, tengo sed y tomo agua..." (V30) }\end{array}$ \\
\hline & $\begin{array}{l}\text { "Entrevistador: ¿y por qué la eliminaron [la leche]? R: por salud también, porque hay estudios } \\
\text { que avalan que la leche no sirve para nada, que al contrario, por ejemplo, mi hija la de } 25 \text {, ella } \\
\text { tiene un problema de procesar los alimentos, la leche, le frena más ese proceso, la leche, el } \\
\text { yogurt, ella se pone estítica, le tiene como alergia a la leche, a los lácteos... entonces nosotros } \\
\text { el único lácteo que usamos es el queso (R48)" }\end{array}$ \\
\hline $\begin{array}{l}\text { Asuntos financieros: "No } \\
\text { tengo dinero suficiente" }\end{array}$ & $\begin{array}{l}\text { "Esta es una imagen de la carnicería de mi barrio, es más barata... tengo un presupuesto, pero } \\
\text { que no me alcanza para todo lo que quisiera... quisiera comprar más carnes, más verduras... } \\
\text { Como ensaladas a lo lejos... El arroz y los fideos son la base de mi alimentación... esto es prin- } \\
\text { cipalmente por la plata [referido a que estos alimentos son más barato]. " (P10) }\end{array}$ \\
\hline \multirow[t]{2}{*}{$\begin{array}{l}\text { Ocasiones especiales: } \\
\text { "Hoy es un día para } \\
\text { celebrar" }\end{array}$} & $\begin{array}{l}\text { "...el asado también se consumió para el año nuevo, pero bien contundente, con cerdo, vacu- } \\
\text { no, longaniza, vienesa... al otro día nos fuimos a la piscina y se comió asado, hamburguesa, } \\
\text { esas cosas... para la pascua se consumió carne a la cacerola con arroz... (E14) }\end{array}$ \\
\hline & $\begin{array}{l}\text { "... [su primo] nos invitó a conocer su casa nueva y obviamente hicieron asado, porque en la } \\
\text { familia el asado no falta..." (B52) }\end{array}$ \\
\hline \multirow{2}{*}{$\begin{array}{l}\text { Disponibilidad de } \\
\text { alimentos: "Si lo tengo } \\
\text { en mi casa o en mi } \\
\text { vecindario" }\end{array}$} & $\begin{array}{l}\text { "Tengo acceso a todo... a todo lo que necesito... Tengo el supermercado de las cosas, la feria } \\
\text { la tengo cerca... todo está disponible..." (B3) }\end{array}$ \\
\hline & $\begin{array}{l}\text { "...aquí tenemos harta feria, lo bueno que tenemos feria el martes, tenemos feria el miércoles, } \\
\text { tenemos feria el jueves y tenemos feria el viernes, tenemos feria casi todos los días... ah! Y la } \\
\text { del domingo" (V33) }\end{array}$ \\
\hline
\end{tabular}

el verano podían encontrar más disponibilidad y diversidad de frutas y verduras en el mercado, comprando y comiendo más.

\section{Percepciones acerca de los alimentos: "Este alimento es..."}

Estas percepciones se asociaron a atributos de alimentos relacionados con condiciones de salud, los que influían en el tipo de producto que preparaban para comer. Cuando ellas percibían que un alimento tenía propiedades saludables, tales como mejorar su digestión o reducir el peso, lo comían frecuentemente. En contraste, ellas describían ciertos alimentos como "enemigos" de su salud en general o en el peso corporal, refiriéndose principalmente al pan y refrescos azucarados, evitándolos.

Estas percepciones se relacionaban al 


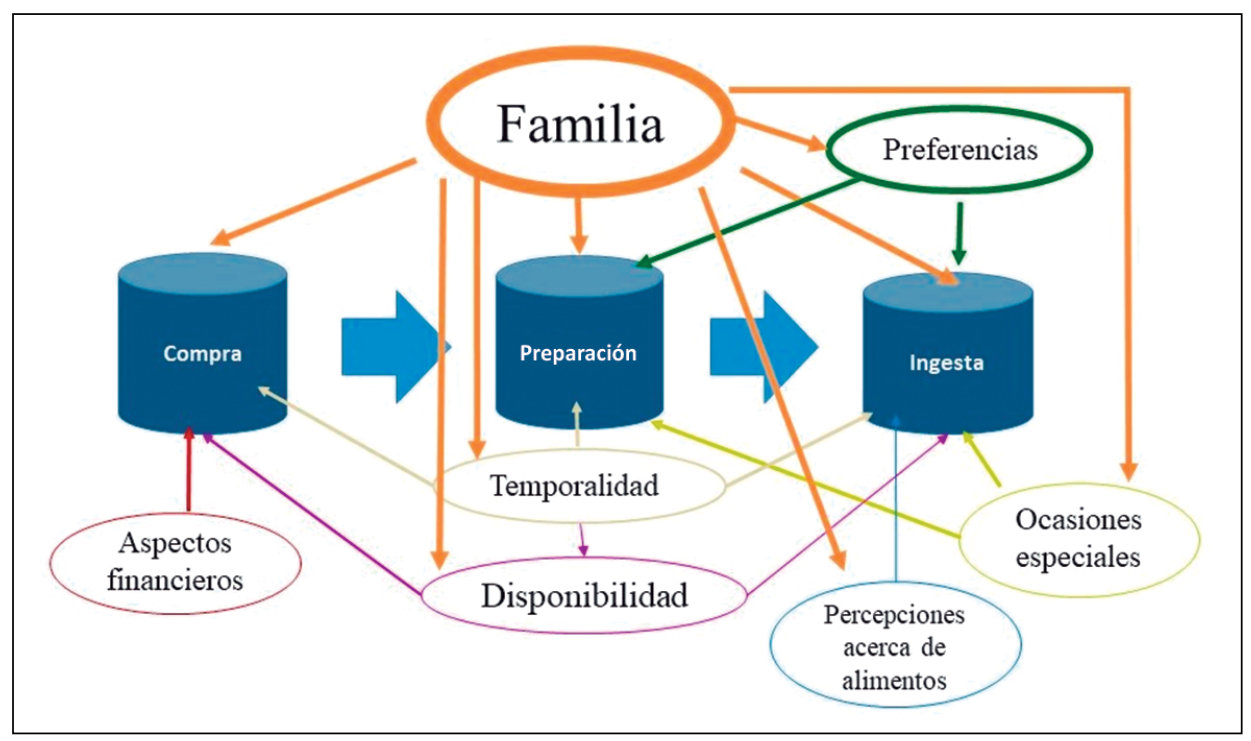

Figura 1.

conocimiento alimentario-nutricional que tenían, el que provenía de familiares, como madres, abuelas o suegras, así como también de páginas web y programas de televisión (particularmente aquellos para amas de casa).

\section{Los asuntos financieros: "No tengo suficiente dinero...”}

Los asuntos financieros fueron descritos como una percepción de no tener dinero o que este era insuficiente para comprar todos los alimentos deseados. La compra de alimentos competía con otros gastos del hogar, tales como el pago de servicios básicos de la casa o las necesidades no alimentarias de sus hijos.

El lugar de compra de alimentos era escogido de acuerdo a donde podían encontrar precios más económicos. Por ejemplo, compraban frutas y verduras en ferias libres, percibiendo estos lugares como los más "baratos" para estos alimentos. Pero, para comprar alimentos como pasta, arroz $\mathrm{o}$ aceite, se dirigían a supermercados, porque percibían que estos alimentos eran más baratos en estos lugares.

\section{Las ocasiones especiales: "Hoy es para celebrar"}

Existen días en los que ellas preparaban comidas especiales $y$, por ende, comían de manera diferente. Estos momentos están relacionados con fechas específicas, como celebraciones de Navidad o Año Nuevo, celebraciones de cumpleaños u otras en las que se desvían absolutamente de su rutina alimentaria tradicional, consumiendo alimentos en mayor cantidad y de mayor densidad calórica. Estas ocasiones especiales eran destacadas como oportunidades en las que la familia, inclusive la extendida, se reunía con las participantes.

\section{La disponibilidad de alimentos: "Si lo tengo en mi casa o en mi vecindario"}

Las participantes otorgaron una doble dimensión a la disponibilidad de alimentos. Primero, hablabron sobre la disponibilidad de alimentos en el hogar, indicando que la presencia o ausencia de ciertos alimentos determinaban su consumo. Tener alimentos para sus hijos (como galletas u otros bocadillos) o lo que sus maridos/ parejas traían, representaba una "tentación” y, por ende, los comían con más frecuencia.

Segundo, si los alimentos estaban disponibles en su vecindario era más probable que los compraran y comieran. En este sentido, la mayoría de las participantes evaluaron su vecindario como un lugar donde podían encontrar la mayoría de los alimentos que necesitaban. Mencionaron tener, a poca distancia de sus hogares, varias tiendas pequeñas de alimentos para comprar lo que necesitan en una emergencia, ferias libres varios días a la semana, y al menos un supermercado.

A su vez, esta disponibilidad se relacionaba con 
la temporalidad, debido a los cambios en la oferta de alimentos en sus vecindarios entre el verano y el invierno.

\section{Discusión}

El presente estudio se realizó con mujeres chilenas viviendo en zonas urbanas vulnerables, en Santiago de Chile. Utilizamos photo-elicitation como un método participativo para recopilar información. Desde las narraciones, se analizó cómo tres conductas alimentarias (compra, preparación e ingesta de alimentos) eran explicadas por siete factores (la familia, preferencias, percepciones sobre alimentos, asuntos financieros, ocasiones especiales, temporalidad y disponibilidad de alimentos). Estos factores pertenecen tanto al intrapersonal como al entorno en el que las participantes se desenvuelven.

Estos factores no solo determinaban de manera diferente las conductas alimentarias, sino que algunos de ellos interactuaban entre sí, para producir un efecto sobre la conducta (Figura 1). Por ejemplo, la familia influenciaba las preferencias o algunas percepciones de los alimentos o la disponibilidad de alimentos era influenciada por la temporalidad. En la literatura sobre conductas alimentarias, se ha sugerido la necesidad de considerar iniciativas multinivel para lograr una alimentación saludable en la población, argumentando que un nivel solamente podría mejorar, si se progresa en otros niveles ${ }^{22,23}$.

Entre los factores que fundamentaban las conductas alimentarias, el centro queda en la familia, por tanto, el posible contexto original del que podría haberse explicado las conductas -la vulnerabilidad o falta de recursos- se ve modificado y complejizado hacia el contexto del ambiente familiar. Esta observación se condice con el concepto de familismo que aparece en la literatura latinoamericana, refiriéndose a la importancia de la familia y a los fuertes vínculos entre los miembros de la familia ${ }^{24}$. En alimentación, Keller, Fleury y Rivera ${ }^{25}$ explican cómo las mujeres cambian sus comportamientos alimentarios cuando sus maridos o hijos no están con ellos, pudiendo comer alimentos menos saludables o prestar más atención a su peso. Otros estudios muestran cómo las participantes priorizan la felicidad de sus hijos en cuanto a la alimentación, por encima de la suya ${ }^{9,26}$. Estos hallazgos demuestran la necesidad de considerar a los miembros familiares en intervenciones destinadas a este grupo de la población.

También llama la atención los cambios de alimentación de las participantes de acuerdo a la época del año, al día de la semana y en momentos especiales. Si bien, estas variables han sido poco estudiadas en el país, internacionalmente se concuerda en que una alimentación menos saludable ocurriría durante épocas más frías ${ }^{27}$, fines de semanas ${ }^{28}$ y eventos sociales ${ }^{29}$. En este sentido, se hacen necesarias estrategias que fomenten el consumo de frutas y verduras anualmente, como podría ser el fomento del uso de las más de 1.000 ferias libres a lo largo del país ${ }^{30}$, lo que, además, contribuiría al presupuesto familiar, o promover el uso de estos alimentos en situaciones de reunión familiar. Por otro lado, el aumento del precio a los alimentos no saludables, como ha sido sugerido recientemente $^{31}$, podría reducir los cambios durante los momentos especiales.

Una limitación de este estudio es que solo incluimos mujeres con familia, por lo cual podrían haberla mencionado como una influencia importante. Además, esta investigación se realizó en verano, lo cual pudo haber influenciado en que la temporalidad apareciera como una variable determinante de las conductas alimentarias, dado que en Chile es notorio el cambio de disponibilidad de ciertos alimentos entre épocas del año. Finalmente, dado que usamos imágenes como base para las entrevistas, estas podrían ser reflejo de lo que ellas pensaron sería bien aceptado por los investigadores.

En conclusión, este estudio contribuye a la comprensión de conductas alimentarias en mujeres chilenas de comunas vulnerables, ofreciendo información útil sobre cómo tres conductas alimentarias son fundamentadas en siete factores. La familia representó uno de los factores más importantes, influyendo en los tres comportamientos estudiados y en otros factores. Incluir a las familias en las intervenciones que promueven una alimentación saludable en las mujeres podría tener más beneficios para ellas, pero también para la familia completa. Además, se necesitan más investigaciones para establecer el impacto de las estaciones en las dietas de los individuos, así como el efecto del fin de semana y ocasiones especiales. Los hallazgos de este 
estudio, orientados a mejorar la comprensión de distintos factores individuales, interpersonales y contextuales, podrían tener un impacto importante en la forma en que promovemos dietas saludables en mujeres más desventajados, aportando con intervenciones culturalmente más aceptadas, que integrarían factores más relevantes para ellas, y que van más allá del nivel individual.

\section{Referencias}

1. FAO, OPS. Panorama de la seguridad alimentaria y nutricional en América Latina y el Caribe [Internet]. Santiago, Chile; 2017. [Consultado en julio de 2018] Disponible en: http://iris.paho.org/xmlui/handle/.

2. Organización de las Naciones Unidas para la Alimentación y la Agricultura, Organizacion Panamericana de la Salud. Panorama de la Seguridad Alimentaria y Nutricional en América Latina y el Caribe 2017 [Internet]. Santiago, Chile; 2017. [Consultado en octubre de 2017] Disponible en: http://www.refworld.org.es/pdfid/.

3. Energici MA, Acosta E, Huaiquimilla M, Borquez F. Feminización de la gordura: Estudio cualitativo en Santiago de Chile. Rev Psicol 2016; 25 (2): 1-17.

4. Ministerio de Salud. Gobierno de Chile. Encuesta Nacional de Salud 2009-2010. Santiago, Chile; 2010. [Consultado en abril de 2018] Disponible en: http:// buenaspracticasaps.cl/wp-content/uploads/.

5. Ministerio de Salud. Gobierno de Chile. Encuesta Nacional de Salud 2016-2017. Primeros resultados [Internet]. Santiago, Chile; 2017. [Consultado en diciembre de 2017] Disponible en: http://web.minsal.cl/wp-content/ uploads/.

6. Byrd-Bredbenner C, Abbot JM, Cussler E. Relationship of social cognitive theory concepts to mothers' dietary intake and BMI. Matern Child Nutr 2011; 7: 241-52.

7. Mosca L, Benjamin EJ, Berra K, Bezanson JL, Dolor RJ, Lloyd-Jones DM, et al. Effectiveness-based guidelines for the prevention of cardiovascular disease in women-2011 update: A Guideline from the American Heart Association. Circulation 2011; 123: 1243-62.

8. Hupkens C, Knibbe H, Drop M. Social class differences in food consumption. The explanatory value of permissiveness and health and cost considerations. Eur J Public Health 2000; 10: 108-13.

9. Inglis $\mathrm{V}$, Ball $\mathrm{K}$, Crawford $\mathrm{D}$. Why do women of low socioeconomic status have poorer dietary behaviours than women of higher socioeconomic status? A qualitative exploration. Appetite 2005; 45: 334-43.

10. Pérez Gil-Romo SE, Vega-García LA, Romero-Juárez
G. Practicas alimentarias de mujeres rurales: una nueva percepcion del cuerpo? Salud Publica Mex 2007; 49 (1): 52-62.

11. Lenz A, Olinto MTA, Dias-da-Costa JS, Alves AL, Balbinotti M, Pattussi MP, et al. Socioeconomic, demographic and lifestyle factors associated with dietary patterns of women living in Southern Brazil. Cad Saude Publica 2009; 25 (6): 1297-306.

12. Pereyra I, Erazo M. Evaluación de la alimentación en mujeres atendidas por el Sistema de Salud Público chileno. Arch Latinoam Nutr 2011; 61: 254-61.

13. Johnson CM, Sharkey JR, McIntosh AW, Dean WR. "I'm the Momma": using photo-elicitation to understand matrilineal influence on family food choice. BMC Womens Health 2010; 10: 21-34.

14. Lachal J, Speranza M, Taïeb O, Falissard B, Lefevvre $\mathrm{H}$, Qualigramh, et al. Qualitative research using photo-elicitation to explore the role of food in family relationships among obese adolescents. Appetite 2012; 58: 1099-105.

15. Lorenz LS, Kolb B. Involving the public through participatory visual research methods. Heal Expect 2009; 12 : 262-74.

16. Krathwohl DR. Qualitative research methods. En: Methods of educational and social science research. Long Grove, IL: Waveland Press, Inc; 2009: 235-58.

17. Secretaría Regional Ministerial de Desarrollo Social- Gobierno de Chile. Índice De Prioridad Social De Comunas 2014 [Internet]. Santiago, Chile; 2014. [Consultado en mayo de 2014]. Disponible en: http://ciperchile.cl/ wp-content/uploads/.

18. Shaffer R. Beyond the dispensary [Internet]. Nairobi, Kenya: the African Medical and Research Foundation; 1983. [Consultado en abril de 2014]. Disponible en: http://www.amoshealth.org/wp-content/uploads/.

19. Galvez EP, Vizcarra M, Palomino AM, Valencia A, Iglesias L, Schwingel A. The photo-elicitation of food worlds: A study on the eating behaviors of low socioeconomic Chilean women. Appetite 2017; 111: 96-104.

20. Saunders B, Sim J, Kingstone T, Baker S, Waterfield J, Bartlam B, et al. Saturation in qualitative research: Exploring its conceptualization and operationalization. Qual Quant 2017; 1-15.

21. Saldaña J. First cycle coding methods. In: The coding manual for qualitative researchers. Thousand Oaks, CA: SAGE Publications Inc., 2013: 58-186.

22. Trapp GSA, Hickling S, Christian HE, Bull F, Timperio AF, Boruff B, et al. Individual, social, and environmental correlates of healthy and unhealthy eating. Heal Educ Behav 2015; 42 (6): 759-68.

23. Hardcastle SJ, Thøgersen-Ntoumani C, Chatzisarantis 
NLD. Food choice and nutrition: A social psychological perspective. Nutrients 2015; 7: 8712-5.

24. Katiria Pérez G, Cruess D. The impact of familism on physical and mental health among Hispanics in the United States. Health Psychol Rev 2014; 8: 95-127.

25. Keller C, Fleury J, Rivera A. Visual methods in the assessment of diet intake in Mexican American women. West J Nurs Res 2007; 29: 758-73.

26. Johnson CM, Sharkey JR, Dean WR, McIntosh A, Kubena KS. It's who I am and what we eat. Mothers' food-related identities in family food choice. Appetite. 2011; 57: 220-8.

27. Prasad M, Lumia M, Erkkola M, Tapanainen H, Kronberg-Kippilä C, Tuokkola J, et al. Diet composition of pregnant Finnish women: Changes over time and across seasons. Public Health Nutr 2010; 13: 939-46.
28. Hart CN, Raynor HA, Osterholt KM, Jelalian E, Wing RR. Eating and activity habits of overweight children on weekdays and weekends. Int J Pediatr Obes 2011; 6: 467-72.

29. Isoldi KK, Dalton S. Calories in the classroom: Celebration foods offered and consumed during classroom parties at an elementary school in a low-income, urban community. Child Obes 2012; 8: 378-83.

30. Ball K, Crawford D, Mishra G. Socio-economic inequalities in women's fruit and vegetable intakes: a multilevel study of individual, social and environmental mediators. Public Health Nutr 2006; 9 (5): 623-30.

31. Siebert F. Académicos apuestan por subir impuesto a alimentos ricos en grasa, sodio y azúcar [Internet]. Santiago, Chile; 2018 [Consultado en abril de 2018]. Disponible en: http://www.uchile.cl/noticias/. 